\title{
Agents Methodologies for e-Chartering Market design
}

\author{
Manolis Sardis, Ilias Maglogiannis \\ University of the Aegean, Department of Information \& Communication \\ Systems Engineering, Samos, Greece, \\ WWW home page: http://www.icsd.aegean.gr \\ emails: sardis@aegean.gr,imaglo@aegean.gr
}

\begin{abstract}
Electronic business and agents are among the most important and exciting areas of research and development in information and communication technology, with considerable potential impact and opportunities for the Maritime sector. This paper proposes the design of a Multi-Agent system for Internet Virtual Chartering Markets (MAVCM). The MAVCM system applies for business-to-business transactions in Maritime markets, and provides mechanisms for Internet-based chartering informational and transactional services. The lifecycle of the proposed system offers a solution for efficiently handling the processes involving a charterer who owns the cargo and employs a shipbroker to find a shipowner to deliver the cargo for a certain freight rate. The objective is to enable Maritime market participants to electronically charter, trade and transport cargos based on information and transactions over Internet via their software agents.
\end{abstract}

\section{Introduction}

Electronic business (e-business) can be defined as the process of sharing business information, maintaining business relationships, and conducting business transactions by means of information and communication technologies [1]. With the advent of Internet-based business-to-business (B2B) electronic markets, real opportunities for online transactions have opened up. The Maritime industry has started to recognize the importance of internet and of the electronic markets. Lower costs and less response time are some of the benefits when compared to the traditional procedures operated by humans. However, the most promising Internetbased electronic markets [3], in Maritime are found in the B2B environment where they act as or intermediaries (middlemen) between ships and cargoes [8], [3]. The agent technology can provide a new way of analyzing, designing, and implementing such electronic markets. The use of software agents has mostly been directed towards applications that support business-to-consumer (B2C) transactions [2].

Please use the following format when citing this chapter:

Sardis, M., Maglogiannis, I., 2007, in IFIP International Federation for Information Processing, Volume 247, Artificial Intelligence and Innovations 2007: From Theory to Applications, eds. Boukis, C., Pnevmatikakis, L., Polymenakos, L., (Boston: Springer), pp. 175-185. 
However, another important domain is the automation of business transactions that occur in B2B electronic markets. Despite the growing number of electronic markets, conducting electronic transactions is still not trivial. Different obstacles exist such as slow response time, lack of implementation between practical and electronic transactions, poor Web business plan and security problems in critical transactions between a seller and a buyer. A lack of explicit legal provisions on B2B e-markets and the (un)fair commercial practices. The result is a lack of transparency in business transactions. According to the literature, it is accepted that agent technology has the ability to address some of these obstacles [3].

Within this context, this paper proposes the design of a multi-agent system of a Virtual Chartering Market (VCM), called Multi-Agent VCM (MAVCM). The MAVCM system is an Internet e-commerce system for B2B transactions in Maritime chartering markets, providing mechanisms for Internet-based chartering of a vessel for cargo transportation, after an e-investigation and an e-negotiation procedure carried on by agents and web based technologies. Our scope is to overcome traditional chartering limitations using the MAVCM platform. However, designing multi-agent based systems is a complex and demanding task, and so far many standardized design methodologies have been established. Some of such methodologies are Gaia [4], MESSAGE [5], TROPOS [6], and ROADMAP [7]. All of these methodologies share the idea that a multi-agent system may be viewed as an organized society of individual agents with their roles and different kinds of interactions among them specified according to specific protocols that are related to the roles of the interacting agents. The new Gaia proposal [4] is specially significant when used for the analysis and design of an open multi agent system. The success of the Unified Modeling Language (UML) in unifying many different object-oriented approaches as well as the fact that Agent UML (AUML) [9], [12], [13] is considered as a natural starting point for modeling in a very rich and expressive way the Agents Interaction Protocols (AIP), that constitute a central aspect for open multi-agent systems, led to the idea of applying AUML to the design of MAVCM. The goal of this paper is to describe the analysis and the design of a global multi-agent eChartering system using multi-agent technologies.

The paper is composed of three parts. In the first part we analyze the business process of the Maritime chartering, the participating actors and their roles. Also the business transactions that occur and their constraints and requirements are presented. In the second part, the information technology solutions based on agent state of the art methodologies and technologies are introduced based on Maritime chartering procedures and requirements. The third part analyzes the design of the e-Chartering system that improves all the traditional methods of the Maritime chartering and brokering procedures giving add-on for the Maritime community using the proposed technologies.

\section{The Charter Market}

The charter market is by no means a unified and homogenous one. It consists of a number of distinct, separate markets, which are neither interdependent nor clearly and sharply set apart. These features favor the creation of diversiform and heterogonous tendencies in the context of the charter market as a whole. According 
to the international bibliography pertaining to the subject as well as to the shipping practice, the charter market is divided in categories based on the following criteria: the type of ship, the type and nature of the bulk to be carried, the geographical distribution of Maritime commercial transportation and the sea areas where ships are certified to operate, the duration of the charter period and the type of charter. The key parameters of every charter are the type of ship and that of cargo.

\subsection{Charter market key players and processes}

The involved actors of the chartering procedure are the following:

1. Shipowner, can be a person or a firm owning one or more ships.

2. Charterer, the owner of the cargo (Cargo owner) is also the representative of the cargo interest in the charter party.

3. Shipbroker, can be a chartering owner's broker, negotiating the terms for the charter of a ship on behalf of a charterer or a shipowner respectively. He can also act as a loading broker whose business is to attract cargoes to the ships of his principal, or as sale and purchase broker, negotiating on behalf of a buyer or seller of a ship.

4. Chartering broker, an intermediate between shipowners with ships available for charter and charterers who wish to charter them to transport cargo in.

\subsection{Communication network between chartering participants}

Global events (financial, political, social etc.) influence the freight rates as well as the position occupied by the company in the field of competition in shipping. Information is exchanged between shipowners, brokers and charterers concerning matters such as ships' and cargo's demand and supply. It is obvious that the quality and the quantity of information exchanged between professionals of the shipping field depend largely on the sort of communication the persons involved have and the according cost/fees they pay for accessing that info. The main resources that bring chartering info are, (1) centers of information concerning the shipping market (Baltic Exhange [8], Bimco, Lloyds of London, etc.), (2) different types and forms of Information (Market reports, Fixture reports, Cargo Orders, Indications, etc.), (3) sources of Information (Chartering negotiations, Maritime institutions, shipping institutes and professional unions, research centers, publishing houses specializing in shipping, the Internet). The 'family' type relationships are an obstacle for medium and small companies to participate with, or almost, the same rules in chartering market.

\subsection{MAVCM requirements based on e-Chartering market}

According to the free market rules (bulk shipping), the procedure until the signing of the chartering market includes the following stages. The stage of investigation, during which the seller, buyer or charterer seeks the appropriate vessel in order to carry the cargo, and the shipowner charterer is looking for the appropriate cargo to carry in his/her ship. At this stage, the charterer and the shipowner make their entry in the market by manifesting their interest which is expressed in the drafting of an "order" and of a "position list". The stage of negotiation features all dealings between the carrier and the cargo owner aiming to conclude a charter party. Finally, the follow up stage, where the charterer and the shipowner have agreed on freight 
rate details and they proceed to carry the cargo by sea or to put the ship at the charter's disposal to that effect. The promise in question is undertaken in exchange for a fee termed freight or hire.

\subsection{Order and Position}

The charterer penetrates the market manifesting his/her interest by putting together an order. The order is drawn up in conformity with the type of chartering in view (voyage charter, consecutive voyage charter, time charter, bareboat charter, charter of affreightment). When the shipowner makes an entry in the market manifesting his/her interest by putting a position. The position usually contains information dealing with the ship type and characteristics, how and where the owner wishes to charter it, the period during which the vessel will be chartered, etc. The key target of the negotiations in chartering is the fixing of the freight rate.

\section{Applying Gaia and A-UML in e-Chartering Market}

The Gaia methodology models multi agents systems from the macro (societal) level to the micro (agent) level. The Gaia methodology [4] comprises three major phases: analysis, architectural design and detailed design. The first phase, analysis, assumes that the application requirements have already been compiled. This phase is concerned with the collection and organization of the system specification. In the architectural design phase, the organizational structure is defined in terms of its topology and regime control, and the interaction model and role model are completed. The last phase, detailed design, is concerned with detailing the agent model, which consists of mapping the identified roles to agent classes and instances, and the services model, which are the blocks of activities in which the agents will engage. The specifications of agents with their roles and the interactions among them and with the environment, are not enough to capture the complex and emergent behavior derived from many self interested agent applications. The complexity of the MAVCM system infrastructure, as stated in section two, needs additional effort for modeling the organizational structure as well as the organizational rules. Agent UML (AUML) builds on the acknowledged success of UML in supporting industrial-strength software engineering. The core part of AUML is the Agent Interaction Protocol (AIP) [30] that constitutes a central aspect for open MAS, specified by means of protocol diagrams. Protocol diagrams extensions to UML include agent roles, multithreaded lifelines, extended message semantics, parameterized nested protocols, and protocol templates. The proposed MAVCM solution for e-Chartering is specified using Gaia methodology and replacing the preliminary interaction model with the AUML notation. The following paragraphs analyze the design considerations of this platform and describe the methodology used.

\subsection{MAVCM requirements}

The initial scope of this architecture is to provide mechanisms for the implementation of the following functions: stage of investigation, stage of 
negotiation and the follow up stage. The structure should be generic enough to suit different supervisory applications, and should provide means for the integration of diverse tools and techniques. An additional requirement is that this architecture provides means for the future expansion of the functions. The FIPA-ACL [14] agent communication language will be the linking language between different implementations of the MAVCM platform, on any FIPA compliant platform. It should be noted that this paper is presenting only the first two main stages (analysis, design). The implementation phase, where different technologically approaches found in literature [17], [18], [19], [20] for MAS systems and electronic markets are under research for the MAVCM platform.

\subsection{The environmental model}

Gaia methodology suggests a general approach to model the MAS environment. This environment is represented in terms of abstract computational resources that can be detected and changed by the agents. In MAVCM, the resources of interest are the vessel information depicted as POSITION and the cargo information depicted as ORDER in "Fig. 1".

\subsection{Organizational structure}

MAVCM is organized by federations, or domains, according to different services provided. The integration of different tools and techniques is achieved through the mapping of their capabilities into the agent roles and by the implementation of agent wrappers. MAVCM domains are organized differently depending on the case. Each agent is responsible for each shipowner or cargowner. Agents of the same port are controlled and organized based on their Broker functionality. The agent organization for each port is controlled by an agent Broker. The Broker is responsible for the manipulation and the agent peer collection in which all members have the same level of authority. The Broker also controls other resources such as Maritime News, forums info, Web Services which for the specific port are important parameters for the investigation phase of the e-Chartering procedure. The internal structure of agents and Brokers is analyzed in more detail in "Fig. 2", where internal

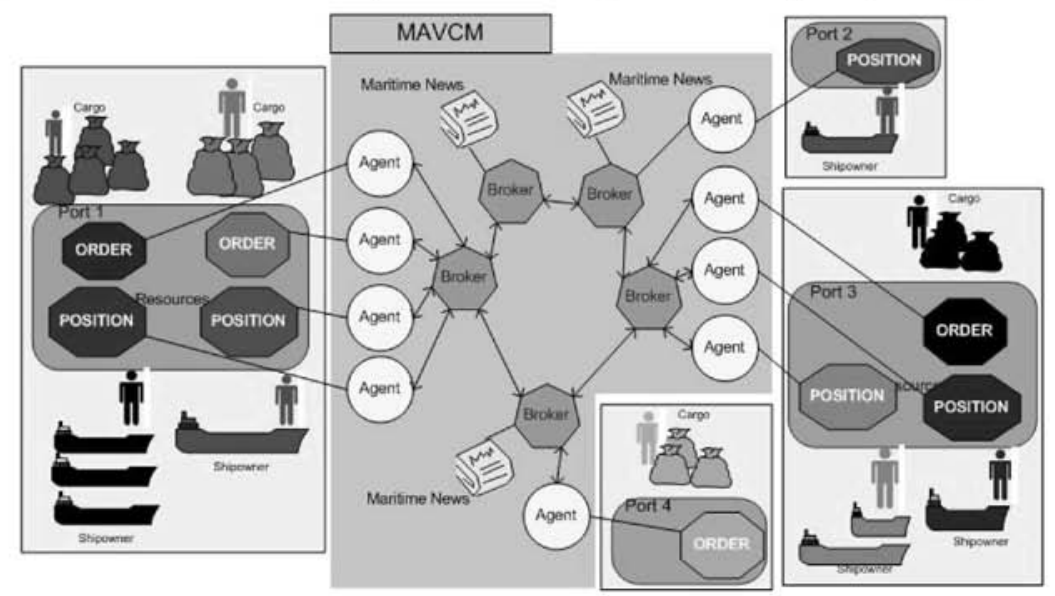

Fig. 1, MAVCM organization structure 
relationships and dependencies are presented. The MAVCM architecture was divided according to the different services provided, grouped by domains. Each service domain, in turn, may comprise other domains. At the highest level of abstraction, the system is sub-divided into six service domains: agent platform services (APS), information services (IS), investigation services (INVS), data collection (DC), negotiation services (NS) and contract services (CS). The APS module is responsible for providing the communication infrastructure with naming and yellow pages services. Contains the agent controller (AC) module, which is responsible for controlling the agent interactions and the process communication with each other in the multi-agent system [10]. The directory facilitator (DF) is a yellow pages service in which agents may register their services (to offer them to other agents), and search for services offered by other agents. Therefore, it has the following functions: register, deregister, modify and search [14]. The IS module is responsible for the storage and retrieval of information when it is required by users and other agents. Also it supports the end user with the user interface (UI) to accomplish the necessary actions for the e-Chartering procedures. The core module for the MAVCM platform is the INVS module, which includes the modules investigation manager (INVM), data analysis (DA) and algorithms for best solution (ABS). The purpose of these modules is to collect and analyze data from the available data sources of the MAVCM platform and then, by analyzing them through the ABS module, to offer the best scenario(s) at the MAVCM registered user. The data collection (DC) module is responsible for the data collection, the data management (DM) module, from the end users ORDERs or POSITIONs and from external resources (yellow pages, Maritime web services, etc.) through the functionality of the external resources management (ERM) module.

When the user has found data scenarios, then the NS module is responsible for the negotiation procedures with the data providers. This module includes the necessary control tools for secure multi-user negotiations (e-auctions) and data exchange with history keeping and user transactions storage capabilities. The behavioral-phase model controller (BPMC) [21] is responsible for the negotiation stages and

\begin{tabular}{|c|c|c|}
\hline \multicolumn{3}{|c|}{ MAVCM AGENT MODULES } \\
\hline $\begin{array}{l}\text { Information Services (IS) } \\
\text { User Interface (UI) } \\
\text { User Database (UD) }\end{array}$ & $\begin{array}{l}\text { Agent Platform } \\
\text { Services (APS) } \\
\text { Agent Controller (AC) } \\
\text { Directory Facilitator (DF) }\end{array}$ & $\begin{array}{c}\frac{\text { Negotiation }}{\text { Services (NS) }} \\
\begin{array}{c}\text { Behavioral - phase } \\
\text { model Controller (BPMC) }\end{array} \\
\text { Protocol Management (PM) }\end{array}$ \\
\hline $\begin{array}{l}\frac{\text { Investigation Services }}{\text { (INVS) }} \\
\text { Investigation Manager (INVM) } \\
\text { Data Analysis (DA) } \\
\text { Algorithms for best } \\
\text { solution (ABS) }\end{array}$ & $\begin{array}{l}\text { Data Collection (DC) } \\
\text { Data management (DM) } \\
\text { External resources } \\
\text { management (ERM) }\end{array}$ & $\begin{array}{c}\frac{\text { Contract }}{\text { Services (CS) }} \\
\text { Contract Management (CM) } \\
\text { Security Controller (SC) }\end{array}$ \\
\hline
\end{tabular}

Fig. 2. MAVCM agent's internal modules 
procedures, by controlling the involved agents interactions and inform the MAVCM user for automatic or semi-automatic status of the negotiation before the finalization of the negotiation. The protocol management (PM) module is controlling the interactions protocol between different agents that are participating in the MAVCM platform. Based on the chartering procedures, the final step for a web user will be implemented using the contact services (CS) module where the final decisions between two negotiated parties will be finalized by the creation of the electronic contract during the follow up stage. This module structure will include all the tools for contract creation and digital signing through the security controller (SC) module responsible for the security part of the contracts.

This MAVCM platform organization provides a balance of flexibility and control to facilitate the inclusion of future functionalities like in the user information (UI) module, where web functionality will inform the user for Maritime cargo changes and opportunities on vessel market.

\subsection{Role model}

Agent roles represent the tasks that the system needs to perform. Each agent in the system may have the capability to perform one or more roles. It is possible, for example, to have one agent performing both the data collection role and the investigation role. Additionally, there may be multiple agents performing the same role, providing redundancy mechanisms and adding flexibility to the system, as for instance, it is possible to instantiate multiple agents for the user interface role, with different views of the system for different purposes when the user is a shipowner or a charterer. According to Gaia, a role can be viewed as an abstract description of an entity's expected function and is defined by four attributes, namely, responsibilities, permissions, activities, and protocols. A role model represents the functions that are expected in the system. For the MAVCM platform twelve roles have been identified: User Interface (UI), User Database (UD), Investigation Manager (INVM), Data Analysis (DA), Algorithms for Best Solution (ABS), Agent Controller (AC), Directory Facilitator (DF), Data Management (DM), External Resources Management (ERM), Behavioral Phase Model Controller (BPMC)/Protocol Management (PM), Contract Management (CM) and Security Controller (SC). "Table 1".

Responsibilities determine functionality and, as such, are perhaps the key attribute associated with a role. Responsibilities are divided into two types: liveness that describe those states of affairs that an agent must bring about, given certain environmental conditions, and safety properties which are invariants. An acceptance state of affairs is maintained across all states of execution. The atomic components of a liveness expression are either activities or protocols. Activities are actions performed by agents that do not require interaction with others. Protocols are actions that involve interactions with others. In order to distinguish between these two types of actions, activities appear underlined in the role model.

Finally, permissions are the 'rights' associated with a role, they identify the resources that are available to that role in order to realize its responsibilities. For example, in the INVM Role the Investigation agent has to search zero or more times for POSITIONs or ORDERs elements in other Brokers agents or agents that belong to the same agent domain and then update the AC state or the agent records in DM. 
Protocols and activities described in the organizational rules are not explicitly mentioned in the role models [15].

Table 1. Role Model Using Gaia

\section{Role Schema: Investigation Management (INVM)}

Description: This role is responsible for performing the search functionality between the Broker agents of MAVCM based on specific user requirements of a shipowner or a cargo owner. Its functionality will be separated first in local domains (eg. same port) and then in more ports' Brokers. Also is responsible to maintain and interact with other Broker agents and perform as an intermediate agent.

Protocols and Activities: SearchBrokers, SearchDomainAgents, UpdateState, UpdateAgentRecords

Permissions: SearchAgentConnected, SearchAgentMetadata, SearchBrokerConnected, SearchBrokerMetadata

\section{Responsibilities}

Liveness: $\mathrm{INVM}=\left(\right.$ SearchBrokers ${ }^{\mathrm{w}}$.UpdateState

SearchDomainAgents ${ }^{\mathrm{w}}$. UpdateAgentRecords)

Safety:

\subsection{Services model}

The Services model represents the services associated with each agent role. The agent role is defined by the list of protocols, activities and responsibilities. Table 2 is presenting the services models for the Investigation Management Role of the MAVCM platform.

Table 2, Services Model

\begin{tabular}{|l|l|l|l|c|}
\hline \multicolumn{2}{|l|}{ Service Schema: Investigation Management Role (INVM) } \\
\hline Service & Inputs & Outputs & Pre- Condition & $\begin{array}{l}\text { Post- } \\
\text { Condition }\end{array}$ \\
\hline SearchBrokers & $\begin{array}{l}\text { Search Criteria for } \\
\text { Broker information }\end{array}$ & Broker list & $\begin{array}{l}\text { Connection with } \\
\text { Brokers }\end{array}$ & True \\
\hline SearchDomainAgents & $\begin{array}{l}\text { Search Criteria for } \\
\text { Domain }\end{array}$ & Agent list & $\begin{array}{l}\text { Connection with } \\
\text { DMs agents }\end{array}$ & True \\
\hline UpdateState & $\begin{array}{l}\text { New state from } \\
\text { investigation procedure }\end{array}$ & $\begin{array}{l}\text { New state } \\
\text { updated }\end{array}$ & $\begin{array}{l}\text { Connection with } \\
\text { ACs agents }\end{array}$ & True \\
\hline UpdateAgentRecords & New record from agents & $\begin{array}{l}\text { New record } \\
\text { updated }\end{array}$ & $\begin{array}{l}\text { Connection with } \\
\text { ACs agents }\end{array}$ & True \\
\hline
\end{tabular}

\subsection{Interaction model}

The Interaction Model consists of a set of protocol definitions. The objective of the Interaction Model is to represent the dependencies and relationships between the various roles in the MAVCM platform by utilizing protocol definitions. More attention is paid to the nature and purpose of the interaction than to the sequence of execution steps. Using the Gaia methodology, one can distinguishes the characteristics that are considered extrinsic in order to minimize the impact of 
changes in the organizational structure and to enable roles to be repeated in systems with different structures. The Manager assumption that will control the INVM module behavior is an example of an extrinsic characteristic. Gaia models have been combined with AUML [16] sequence diagrams for the additional detailing of agent interactions.

\subsection{Agent model}

The specification of the agents in MAVCM depends on the tools that compose the solution. Each tool has different capabilities that are associated with different agent roles, thus the definition of the agent model can only be further detailed when the MAVCM conceptual architecture is realized into implementation architecture. For the MAVCM prototype implementation, each role will be mapped to one agent class. This mapping results in a simple agent model with a total of thirteen agent classes as represented in Table 3.

Table 3. Agent model

\begin{tabular}{|l|l|l|}
\hline Role & Class & Instances \\
\hline UI & User Interface & Zero or more \\
\hline UD & User Database & Zero or more \\
\hline INVM & Investigation Manager & One or more \\
\hline DA & Data Analysis & One \\
\hline ABS & Algorithms for Best Solution & Zero or more \\
\hline AC & Agent Controller & One \\
\hline DF & Directory Facilitator & One \\
\hline DM & Data Management & Zero or more \\
\hline EDM & External Resources Management & Zero or more \\
\hline BPMC & Behavior Phase Model Controller & One or more \\
\hline PM & Protocol Management & One or more \\
\hline CM & Contract Management & One or more \\
\hline SC & Security Controller & One or more \\
\hline
\end{tabular}

Our aims were to analyze and design a system that could transform the 'human' chartering procedure into a dynamic and electronic automated system that could implement as much as possible of the human procedures and methodologies that humans perform during the Maritime chartering. This proposed solution gives addon benefits to chartering procedure as it vastly increases customer reach and visibility. Promotes new relationships in a cost-effective fashion. Allows saving time finding quality service cargo providers and shippers. Competitive negotiation promotes market-driven pricing to save money and create healthy e-negotiation transactions. Utilizes the internet to allow Shipowners and Cargo owners to negotiate cargo shipments and available service across any geography and any mode of transport. 


\section{Conclusions}

The organization of MAVCM into functional domains and roles provides a clear coordination of agents inside its domains and facilitates the integration of additional functionalities such as security issues and management of more than one community of MAVCM systems. Our aim was to design an architecture based on Gaia methodologies and AUML that first will describe with an analytic way the chartering human procedures and second to enable the integration of different tools and functionalities that will describe in more detail chartering procedures for specific vessels and cargo types. The main contribution of this research is the transformation of the chartering Maritime market procedures, into an electronic format, where until now most of them are done manually by humans. The identification of the chartering procedures and necessary functions and then the design of a system based on these market requirements using information technology tools over the web is an add-on. The actors of the e-Chartering procedures will be implemented by multi-agents that support the creation of virtual chartering markets, electronic negotiations and the construction of electronic contracts between a shipowner and a cargo owner. The implementation of the proposed system is under research. Negotiation and e-auctions procedures and techniques will be analyzed in more detail. Agent software development framework suites like the JADE [10] platform, and SALSA [11] framework will be evaluated for the implementation of the MAVCM platform.

\section{References}

1. Zwass, V. (1996). Electronic commerce: Structures and issues. International Journal of Electronic Commerce vol. 1(1), 3-23.

2. He, M. and Leung, H. (2002). Agents in e-commerce: State of the art. Knowledge and Information Systems vol. 4, 257-282.

3. Maamar, Z. (2002). Association of users with software agents in e-commerce. Electronic Commerce Research and Applications vol. 1, 104-112.

4. Zambonelli, F., Jennings, N., and Woolddridge, M., "Developing Multiagent Systems. The Gaia Methodology". ACM Transactions on Software Engineering and Methodology, Vol 12, No 3, (pp. 417-470), July 2003.

5. Caire, C., Garrijo, F., Gomez, J., Pavon, J., Leal, F., et al., "Agent oriented analysis using MESSAGE/UML". Proceedings of Agent-Oriented Software Engineering -AOSE 01, Canada, May, 2001.

6. Guinchiglia, F., Mylopoulos, J., and Perini A., "The Tropos Software Development Methodology: Processes, Models and Diagrams", Proceedings of Agent-Oriented Software Engineering (AOSE-2002),Bologna, Italy, July 2002.

7. Juan, T., Pearce, A. and Sterling, L., "ROADMAP: Extending the Gaia Methodology for Complex Open Systems". Proceedings of Autonomous Agents and Multi-Agent Systems - AAMAS '02 (pp. 3-10), Bologna, Italy, July 15-19, 2002.

8. Baltic Exchange, available from http://www.balticexchange.com

9. AUML Home Page, available from http://www.auml.org

10. JADE Home Page, 2004, available from http://jade.tilab.com 
11. Marcela Rodriguez, Jesus Favela, "A Framework for Supporting Autonomous Agents in Ubiquitous Computing Environments", Book Autonomous Agents for Ubiquitous Collaborative Environments, Springer Berlin / Heidelberg, Volume 2313/2002, 2002

12. J. Odell, V. D. Panunak, and B. Bauer. "Extending UML for Agents". In: Wagner, G.,Lesperance, $Y$. and $\mathrm{Yu}, \mathrm{E}$. Proceedings of the Second Workshop on agent-Oriented Information systems (AOIS'00), iCue Publishing, pp. 3-17, 2000.

13. J. Odell, V. D. Parunak, and B. Bauer. "Representing Agent Interaction Protocols in UML ", In Proceedings of the First International Workshop on Agent-Oriented Software Engineering (AOSE'01), Springer Verlag, 121-140.

14. FIPA (Foundation for Intelligent Physical Agents), http://www.fipa.org.

15. Chengqi Zhang, Chunsheng Li, 'An Agent-Based Framework for Petroleum Information Services from Distributed Heterogeneous Data Resources', (APSEC'02), IEEE, 2002.

16. M. Huget, "Agent UML Notation for Multiagent System Design", IEEE Internet Computing, 2004.http://www.fipa.org/specs/fipa00029/SC00029H.html

17. Michael Berger, Steffen Rusitschka, Dimitri Toropov, Michael Watzke, Marc Schlichte, "Porting Agents to Small Mobile Devices-The Development of the Lightweight Extensible Agent Platform", 2003.

18. F. Bellifemine, A. Poggi, G. Rimassa, "Developing multi agent systems with a FIPAcompliant agent framework". In Software Practice \& Experience", John Wiley \& Sons, Ltd. vol no. 31, pp. 103-128, Feb. 2001.

19. Youyong Zou, Tim Finin, Li Ding, Harry Chen, Rong Pan, "Using Semantic Web technology in Multi-Agent Systems: a case study in the TAGA trading agent environment", In Proceedings of the $5^{\text {th }}$ international Conference on Electronic Commerce (Pittsburgh, Pennsylvania, September 30- October 03, 2003). ICEC '03, vol. 50. ACM Press, New York, NY, 95-101.

20. J.M. Vidal, P. Buhler, and C. Stahl, "Multiagent systems with workflows", IEEE Internet Computing, 8(1):76-82, January/ February 2004.

21. Jing Han, Ming Li , Lei Guo, "Soft Control on Collective Behavior of a Group of Autonomous Agents By a Shill Agent", Journal of Systems Science and Complexity, Springer Boston, Vol 19, Number 1 / March, 2006, Pages54-62. 